\section{New antituberculosis drugs: from clinical trial to programmatic use}

\author{
Gina Gualano, ${ }^{1}$ Susanna Capone, ${ }^{2}$ \\ Alberto Matteelli, ${ }^{2}$ Fabrizio Palmieri ${ }^{1}$ \\ 'Respiratory Infectious Diseases Unit, \\ National Institute for Infectious Diseases \\ L. Spallanzani, Rome; ${ }^{2}$ Department of \\ Infectious and Tropical Diseases, WHO \\ Collaborating Centre for TB/HIV and TB \\ Elimination, University of Brescia, Italy
}

\section{Abstract}

Treatment of multidrug-resistant tuberculosis (MDR-TB) cases is challenging because it relies on second-line drugs that are less potent and more toxic than those used in the clinical management of drug-susceptible TB. Moreover, treatment outcomes for MDR-TB are generally poor compared to drug sensitive disease, highlighting the need for of new drugs. For the first time in more than 50 years, two new anti-TB drugs were approved and released. Bedaquiline is a first-in-class diarylquinoline compound that showed durable culture conversion at 24 weeks in phase IIb trials. Delamanid is the first drug of the nitroimidazole class to enter clinical practice. Similarly to bedaquiline results of phase IIb studies showed increased sputum-culture conversion at 2 months and better final treatment outcomes in patients with MDR-TB. Among repurposed drugs linezolid and carbapenems may represent a valuable drug to treat cases of MDR and extensively drugresistant TB. The recommended regimen for MDR-TB is the combination of at least four drugs to which $M$. tuberculosis is likely to be susceptible for the duration of 20 months. Drugs are chosen with a stepwise selection process through five groups on the basis of efficacy, safety, and cost. Clinical phase III trials on new regimen are ongoing that could prove transformative against MDR-TB, by being shorter (six months), simpler (an alloral regimen) and safer than current standard therapy. It is fundamental that the adoption of the new drugs is done responsibly to avoid inappropriate use. Concentration of inpatient MDR-TB treatment in specialized centers could be considered in countries with low numbers of cases in order to provide appropriate clinical case management and to prevent emergence of drug resistance.

\section{New antituberculosis drugs and regimens}

\section{New drugs}

For the first time in more than 50 years, two new anti-tuberculosisi (TB) drugs were approved and released in 2012 and 2013. Bedaquiline is a first-in-class diarylquinoline compound with a novel mechanism of action, the inhibition of bacterial ATP synthase, and potent activity against drug-sensitive and drug resistant $M$. tuberculosis. Registration was granted based on the results of a phase IIb clinical trial, ${ }^{1,2}$ while a phase III trials is still ongoing and results are pending. In the stage II of the phase II registration trial bedaquiline showed durable culture conversion at 24 weeks associated with a high likelihood of response at 120 weeks. $^{3}$ There are unresolved safety concerns with bedaquiline, related to QTc prolongation caused by the drug and the observed and yet unexplained higher mortality rate detected in the group treated with bedaquiline compared to placebo. ${ }^{3}$ However, excess mortality was not observed in other case series of small number of patients. ${ }^{4,5}$ A new phase 3 trial to investigate the safety and efficacy of bedaquiline when used in combination with short multidrug-resistant tuberculosis (MDRTB) regimens of 9 and 6 months' duration respectively is ready to start in the context of the STREAM trial. ${ }^{6}$ Two bedaquiline-containing arms will be tested: group $\mathrm{C}$ is a 9-month oral-only regimen in which bedaquiline replaces kanamycin, and group D is a shortened simplified 6-month regimen containing bedaquiline.

Delamanid is the first drug of the nitroimidazole class to enter clinical practice. The mechanism of action is innovative, as the drug inhibits the synthesis of a cell-wall component of M.tuberculosis. Similarly to bedaquiline, registration was grated following publication of the results of phase IIb studies showing increased sputum-culture conversion at 2 months, ${ }^{7}$ and better final treatment outcomes in patients with MDR-TB (defined as in vitro resistance to at least isoniazid and rifampicin, the two most potent first-line antituberculosis drugs) ${ }^{8,9}$ As for Bedaquiline, QT prolongation is documented, but appears to be of little clinical significance, and the rate of adverse events is similar to those of patients receiving placebo. ${ }^{7}$ A phase III trial on delamanid is ongoing: in this trial the drug is given in combination with moxifloxacin and HIV-positive patients on antiretroviral therapy are included. Two additional ongoing trials are assessing the use of delamanid for treatment of pediatric multidrug-resistant tuberculosis (MDR-TB).

To ensure strict vigilance on the use of new drugs and to prevent emergence of resistance,
Correspondence: Gina Gualano, National Institute for Infectious Diseases Lazzaro Spallanzani, Via Portuense 292, 00149 Rome, Italy.

Tel.: +39.06.55170463 - Fax: +39.06 .55170413 .

E-mail: gina.gualano@inmi.it

Key words: Multidrug-resistant; tuberculosis; antituberculosis therapy; new drugs.

Contributions: GG designed the content of the review and wrote the manuscript; $\mathrm{FP}, \mathrm{AM}$ and $\mathrm{SC}$ wrote the manuscript; FP and AM critically revised the paper.

Conflict of interests: the authors declare no potential conflict of interests.

Received for publication: 29 April 2016. Accepted for publication: 29 April 2016.

This work is licensed under a Creative Commons Attribution-NonCommercial 4.0 International License (CC BY-NC 4.0).

(C) Copyright G. Gualano et al., 2016

Licensee PAGEPress, Italy

Infectious Disease Reports 2016; 8:6569

doi:10.4081/idr.2016.6569

both bedaquiline and delamanid should only be prescribed as part of a World Health Organization (WHO)-recommended regimen for patients with MDR-TB who have documented evidence of resistance to any fluoroquinolones, i.e., a very close proxy of extensively drug-resistant TB (XDR-TB; defined as in vitro resistance to isoniazid and rifampicin plus any fluoroquinolone and at least one of the second-line injectable drugs: amikacin, capreomycin or kanamycin). ${ }^{10,11}$

There are two main unresolved concerns with the two new drugs. In very difficult-totreat patients, it would be ideal to use both in the same patient: however, in the absence of data from clinical trials on their concomitant use, clinicians should be very cautious. ${ }^{12}$ Second, at the end of 2015 access to the two new drugs in high burden countries is still limited. ${ }^{13}$ One of the main challenges in the near future will be to ensure their available to all those in need while carefully adhering to WHO policies for the introduction of new drugs in control programs. ${ }^{14}$

\section{Repurposed drugs}

Among repurposed drugs for the treatment of MDR-TB, clofazimine deserves special attention. Improved treatment success rate, accelerated sputum culture conversion, and accelerated cavity closure was demonstrated in the treatment arm containing clofazimine in a randomized controlled trial. ${ }^{15}$ On the other hand, early bactericidal activity of clofazimine was not encouraging in another experimental 
regimen in combination with new drugs,${ }^{16}$ showing that more data is required.

Linezolid, an oxazolidinone antibiotic, is more and more recognized as an important antituberculosis drug for the treatment of MDR-TB. ${ }^{17,18}$ In a randomized controlled trial of culture-positive pulmonary XDR-TB cases, patients receiving linezolid in addition to an optimized regimen had a significantly higher probability of sputum culture conversions and overall success. ${ }^{19} \mathrm{An}$ important limitation of linezolid is the frequent occurrence of side effects (myelosuppression and neuropathy): however, incidence is limited, while maintaining efficacy, by reducing the dosage from 1200 $\mathrm{mg}$ to $600 \mathrm{mg}$ daily.

Another class of antibacterial agents, the carbapenems, shows promising efficacy and tolerability, in combination with clavulanate, for the treatment of MDR-TB. Studies are limited to case series, and concern meropenem/clavulanate, ${ }^{20}$ and ertapenem. ${ }^{21}$

\section{New regimens}

Important lessons on planning of trials on new drugs and new regimens were learnt while trying to demonstrate that a standard regimen containing a fluoroquinolone could allow shortening treatment of uncomplicated, smear positive pulmonary TB from six to four months. It took ten years and impressive financial commitment to achieve finally disappointing results. Early-phase animal and phase 2 studies gave promising results that were not confirmed in three large,,$^{22,23}$ multi-country, non-inferiority phase 3 clinical trials. ${ }^{24-26}$ Noninferiority of fluoroquinolone-containing regimens compared to the standard regimen was not demonstrated in terms of treatment failure or relapse within 18-24 months after randomization, despite the fact that, in all three trials, observed culture conversion rates at 2 months were within the predicted confidence intervals of the phase 2 data.

This experience shows that better early predictors of final treatment success need to be developed and the design and selection of future experimental regimens will need to incorporate a triage process that can mitigate risks while enabling the accelerated assessment of new drugs and regimens.

A new regimen looks particularly attractive in these days to improve the treatment of drugsensitive and MDR-TB: it consists of pretomanid (previously known as PA-824), moxifloxacin and pyrazinamide (PaMZ). The regimen could prove transformative against MDRTB, by being shorter (six months), simpler (an all-oral regimen) and safer than current standard therapy. It would also reduce dramatically the costs of treatment for MDR-TB. Since preexisting resistance to the three drugs is limited, it is estimated that one-third to one-half of all MDR-TB cases would be sensitive to the three drugs and would be cured by the regimen.

The trial that paved the way for further testing of the PaMZ regimen is an example of an innovative approach to investigate new TB drugs and regimens. ${ }^{27}$ In this short-duration trial, PaMZ proved to be significantly better than bedaquiline alone, bedaquiline plus pyrazinamide, bedaquiline plus pretomanid, and comparable to the standard treatment regimen against drug-sensitive TB. In one single trial several drugs and drug combinations were tested at the same time, allowing to select, for further development, only the most promising combination of drugs (including new drugs).

Eventually, the PaMZ regimen entered the phase $2 b$, with a study of bactericidal activity defined as the decrease in colony forming units (CFUs) of $M$. tuberculosis in the sputum of patients with microscopy smear-positive pulmonary tuberculosis - at eight sites in South Africa and Tanzania. Results showed PaMZ to be safe, well tolerated, and with superior bactericidal activity in drug-susceptible tuberculosis during 8 weeks of treatment. Results were consistent between drug-susceptible and MDR tuberculosis. ${ }^{28}$ Based on these data, TB Alliance and its partners have started in 2015 the STAND (Shortening Treatments by Advancing Novel Drugs) study, a global Phase 3 clinical trial of PaMZ. Researchers expect to enroll 1500 patients in approximately 50 study sites in Africa, Asia, Caribbean, Eastern Europe, and Latin America. MDR-TB patients will receive treatment for 6 months and will be followed for two years starting from the beginning of treatment.

Another pivotal Phase 3 Study, the Nix-TB trial, will evaluate, in an open design, the efficacy, safety, tolerability and pharmacokinetics of 6 months of treatment with bedaquiline plus pretonamid plus linezolid in patients with XDR-TB, or who have treatment intolerant or non-responsive MDR-TB. The trial, launched in March 2015, is conducted by a partnership between TB Alliance, Janssen Pharmaceutica and the sites in South Africa where the study is conducted. Incidence of bacteriologic failure or relapse or clinical failure will be measured through follow up until 24 months after the end of treatment. The trial capitalizes on the availability of novel drugs by studying them together in the most vulnerable TB population, to maximize their impact on the epidemic.

\section{Principles of multidrug-resis- tant tuberculosis treatment}

Treatment of MDR-TB cases is challenging because it relies on drugs that are less potent and more toxic than those used in the clinical management of TB disease caused by drug- susceptible (DS-TB) or MDR strains. ${ }^{29}$ As for susceptible TB forms, MDR-TB should be treated with a combination of drugs in order to cure the patient and avoid relapses, but unfortunately treatment outcomes for MDR-TB are generally poor compared to drug sensitive disease. ${ }^{30,31}$ Recently, new guidelines have been published. ${ }^{32,33}$ Unfortunately, existing data on MDR-TB treatment come mainly from retrospective cohort analyses, so recommendations included in the updated guidelines relies on an evidence of low to very low quality. ${ }^{30,31,34}$

Drug susceptibility testing (DST) (rapid and/or conventional) is strongly recommended by WHO in all cases, since individualized treatment was associated with an improvement in treatment outcomes. ${ }^{32}$ While awaiting second line DST results or in context DST is not available, choice of drug should rely on the DST of the strain isolated from the patient or close contacts with MDR-TB, previous use of the drug in the patient, and the frequency of its use or documented background drug resistance in the setting. Use of drugs to which the strain was reportedly susceptible showed some added benefit, so regimen should be adapted according to culture DST as soon as possible. ${ }^{35}$

Based on their effectiveness and safety, WHO identified five groups of anti-TB drugs. In MDR TB, WHO recommend a combination of at least four drugs, chosen in a hierarchical order (Table 1), to which the M. tuberculosis isolate is thought to be susceptible plus pyrazinamide. ${ }^{32}$ Although there is no evidence supporting the use of more than four drugs in patients with extensive disease, recent data suggest that the use of at least six drugs in the intensive phase could be associated with better treatment success. ${ }^{36}$ It is important to underline the uncertainties around the reproducibility and reliability of DST for pyrazinamide (and ethambutol) as well as the second line anti-TB drugs other than the parenteral agents and the fluoroquinolones. ${ }^{37}$

\section{Designing a regimen to treat mul- tidrug-resistant tuberculosis}

The first step in designing the regimen is to select any Group 1 drugs that are likely to be effective. As noted previously, pyrazinamide is recommended in all regimens, throughout the course of MDR-TB therapy regardless of DST results. Ethambutol should be considered when the patient has not used the drug previously, has failed a WHO category 1 regimen, or the prevalence of resistance is known to be low. ${ }^{38}$ High-dose isoniazid could be a reasonable recommendation, but further studies are needed. In a randomized study evaluating the impact of high-dose isoniazid (16 to $18 \mathrm{mg} / \mathrm{kg}$ ) standard-dose isoniazid ( $5 \mathrm{mg} / \mathrm{kg}$ ), patients who received high-dose isoniazid were more likely to achieve sputum culture conversion and radiographic improvement at 6 months. ${ }^{39}$ 
As second step an injectable agent (WHO Group 2) should be selected. Group 2 drugs include the aminoglycosides (kanamycin, and amikacin) and polypeptides (capreomycin and viomycin) with bactericidal activity. WHO recommends that all patients with MDR/XDR-TB receive an injectable agent as part of their initial therapy. In the systematic review that led to the WHO recommendations, no second-line parenteral agent was found to be superior to another. When choosing an injectable it is important to consider costs, the frequency of adverse events (AE) reactions, and the possibility of cross-resistance. Given its lower cost, WHO suggest kanamycin as first choice with possible amikacin use instead of kanamycin. Streptomycin is not currently recommended for treatment of MDR- and XDR-TB. ${ }^{40,41}$ Fluoroquinolones should always be used unless there is a contraindication. They showed a significant association with cure and this effect was more pronounced in later-generation fluoroquinolones (levofloxacin, moxifloxacin, gatifloxacin and sparfloxacin), and was highest when used against strains known to be susceptible. ${ }^{42}$ Fluoroquinolones are known to prolong the QT interval. QT interval prolongation predisposes to torsades de pointes, which may result in sudden death. There is variability between the fluoroquinolones in this effect; however, the prolon- gation is considered minimal. Additional cardiac monitoring is required when used with drugs that prolong the QT interval (bedaquiline, delamanid, clarithromycin, clofazimine). ${ }^{43,44}$ Group 4 drugs are added until the regimen contains at least four likely effective drugs. Ethionamide or prothionamide should be included in a regimen unless there is a particular contraindication. PAS has shown low efficacy so its use is recommended only if an additional drug is needed to have at least four effective second line drugs in the regimen. ${ }^{45-47}$ The drugs belonging to group 5 should be included whenever four active drugs are not available from previous groups. ${ }^{48,49}$

WHO recommends an intensive phase of 8 months for most patients. When using injectables, the minimum duration of the intensive phase is 8 months, the continuation phase lasting for 12-18 months, for a total treatment duration of at least 20 months. WHO recommends that treatment response be assessed by monthly sputum smear and culture rather than smear microscopy alone, since less frequent monitoring would delay detection of treatment failure.$^{32}$ As a rule, 18 months need to be added to the date of the first negative culture to define the final treatment duration. In the case of failure to achieve culture conversion, the underlying causes (incorrect drug dosage, non adherence factors, malabsorption, drug inter- actions) need to be identified and, possibly, corrected. Determination of the optimal duration of the intensive phase is a high-priority research question because it is likely that patients who convert cultures early in the course of therapy could be treated with a shorter intensive phase ${ }^{33}$ Extension of therapy to 24 months may be indicated in chronic cases with extensive disease.

$\mathrm{AE}$ are very common during the course of treatment, and the most common drug-related $\mathrm{AE}$ reported are listed in Table 2 Patients should be educated about possible side effects and be queried using a standardized approach at each clinical evaluation. Relevant baseline clinical and laboratory examinations should be performed and repeated periodically during the course of therapy. Examples of monitoring algorithms have been published. ${ }^{50}$ Drug-related side effects should be managed promptly and definitively. Mild AEs may be managed symptomatically. Ensuring that the patient understands the importance of each medication, the possible side effects of medication they are taking and what to do when they occur can reduce the risk of serious adverse events..$^{33}$

\section{Short course regimens for mul- tidrug-resistant tuberculosis}

A series of cohort studies conducted in Bangladesh identified a regimens having a

Table 1. World Health Organization groups of first- and second-line anti-tuberculosis drugs.

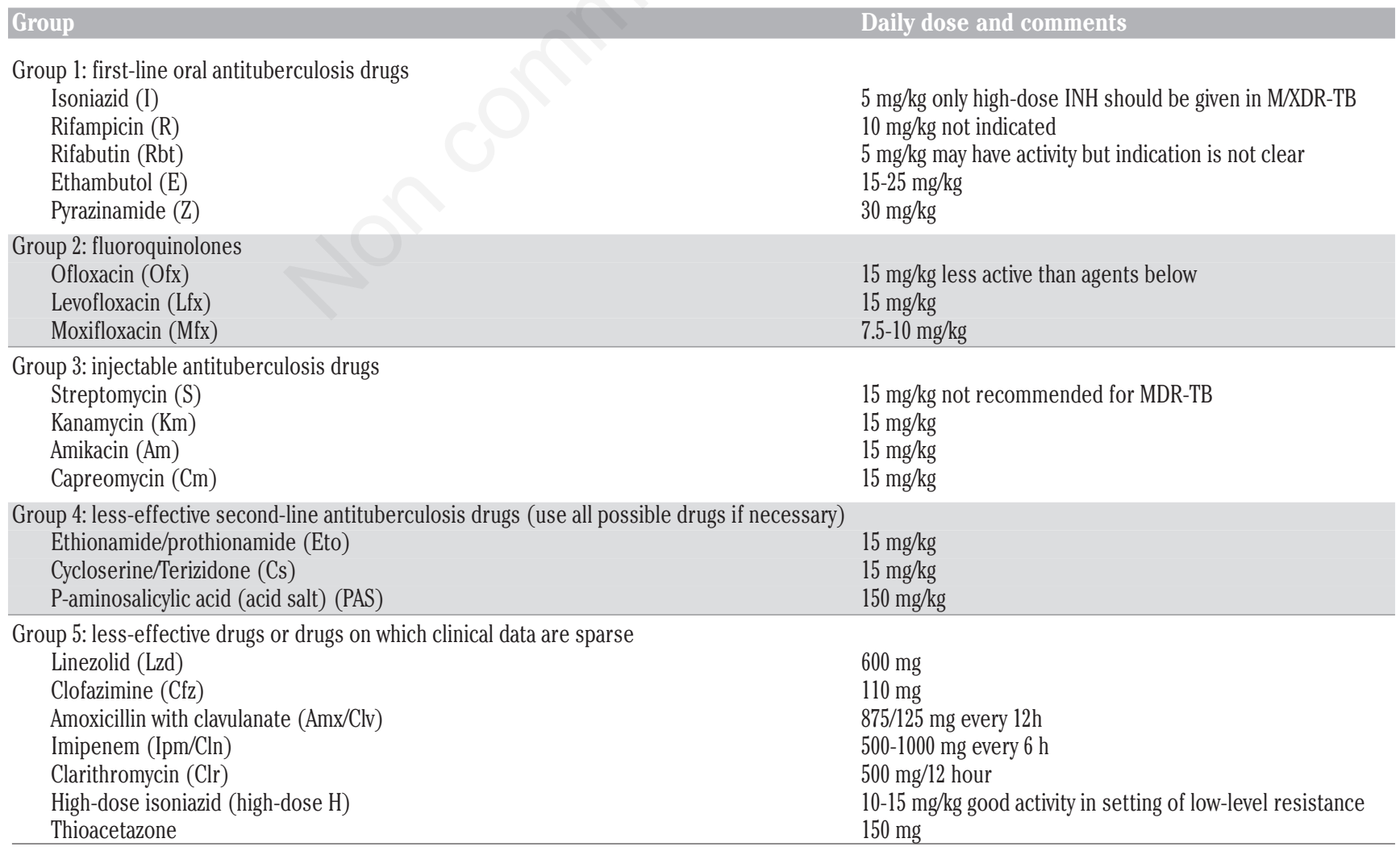


duration of 12 months or less with very promising results in terms of tolerability, completions and cure rate, ${ }^{51}$ compared to the much longer regimen recommended by the WHO for the treatment of MDR-TB. These data were further supported by the results of observational cohorts in Africa. ${ }^{52,53}$ However, there is much less evidence on the effectiveness and safety of these so-called short-regimens compared with regimens lasting 20 months.

WHO recommends that short regimens should only be used within the context of research and under close monitoring for a period of at least 12 months beyond the end of treatment, to early identify those patients who may have a high risk of relapse and acquired resistance. A proper evaluation of the shorter regimen, has been planned by IUATLD in collaboration with the UK Medical Research Council. In a multicenter randomized trial of non-inferiority design a nine-month regimen will be compared to the treatment currently recommended by the World Health Organization in patients with MDR pulmonary TB with no evidence on line probe assay of fluoroquinolone or kanamycin resistance (the STREAM trial). ${ }^{6}$ The study, which is not yet open for participant recruitment, will enroll at least 1155 participants with MDR-TB and will follow them up for a total of 132 weeks. Results from the STREAM trial together with data from ongoing cohorts should provide the evidence necessary to revise current recommendations for the treatment for MDR-TB.

\section{Models of care and healthcare system}

\section{Models of care for managing mul- tidrug-resistant tuberculosis}

In the absence of randomized trials assessing models of care for the programmatic management of MDR-TB, systematic reviews have analyzed evidence on the effectiveness of the ambulatory as compared with hospital-based management of the intensive phase of MDRTB treatment from a wider range of settings. ${ }^{54,55}$ WHO recommends that, where possible, patients with MDR-TB should be treated using ambulatory or community-based care rather than models of care based principally on hospitalization. ${ }^{32}$ National policies and practices differ in the predominant model of care that is employed. The choice between hospitalization and ambulatory treatment depends on several factors in addition to the severity of the disease. Such factors include the availability of hospital beds with adequate infection control measures to prevent nosocomial transmission; the availability of trained personnel at hospitals and clinics to administer treatment and manage adverse drug reactions; the availability of a social support network to facilitate adherence to ambulatory treatment, and provisions for backup facility to manage patients who need inpatient treatment care. Although data available showed a higher proportion of patients achieving treatment success in hospital-based MDR-TB management compared with ambulatory treatment, the difference was not statistically significant. The overall reported adjusted pooled treatment success rate (62-66.4\%) in those reviews was lower than the target of $75 \%$ defined by WHO. Moreover cost-effectiveness of care for a patient receiving treatment for MDR-TB could be improved with an ambulatory model.

The introduction of new drugs offers an opportunity of advancing towards the elimination of TB but the adoption of the new drugs and regimens must be done responsibly to avoid inappropriate use. ${ }^{56}$ If proper patient management is not in place, the risk for development of resistance to the new drugs out-

Table 2. Common adverse effects of drugs used for the treatment of extensive- and multidrug-resistant tuberculosis and their management.

\begin{tabular}{|c|c|c|}
\hline Substance & Common adverse effects & Management \\
\hline \multicolumn{3}{|r|}{ Group I } \\
\hline \multirow{3}{*}{$\begin{array}{l}\text { Ethambutol } \\
\text { Pyrazinamide }\end{array}$} & Optic neuropathy & $\begin{array}{l}\text { Inform the patient to report decreased vision immediately. } \\
\text { Discontinue and refer to an ophthalmologist if vision deteriorates }\end{array}$ \\
\hline & Hepatotoxicity, rash, gout & $\begin{array}{l}\text { Discontinue drug if hepatotoxicity occurs. } \\
\text { For Rash manage symptomatically, if extensive stop drug and consider reintroduction }\end{array}$ \\
\hline & & Group II \\
\hline $\begin{array}{l}\text { Amikacin; Capreomycin; } \\
\text { Kanamycin }\end{array}$ & Ototoxicity, nephrotoxicity & $\begin{array}{l}\text { Monitor levels, hearing and renal function monthly. } \\
\text { If problems occur consider reducing dose frequency to three times a week. } \\
\text { Discontinue if problems persist, but balance risk of cure versus deafness. }\end{array}$ \\
\hline & & Group III \\
\hline
\end{tabular}

Levofloxacin; Moxifloxacin GI disturbances, tendinitis, insomnia QT interval prolongation may be potentiated with other drugs Group IV

Para-Aminosalicylic Acid $\quad$ Nausea and vomiting, gastritis, G Give antiemetics. Hypothyroidism: levotiroxine hepatotoxicity, hypothyroidism

Protionamide/Etionamide $\quad$ GI disturbances, depression, $\quad$ GI disturbances: initiate a stepwise approach to manage nausea and vomiting. hepatotoxicity, hypothyroidism Start antiemetics.

Terizidone/Cycloserine $\quad$ Neurotoxocity, peripheral neuropathy Give high dose pyridoxine, up to $50 \mathrm{mg}$ for every $250 \mathrm{mg}$ of drug.

If neuropathy progresses discontinue drug. Discontinue if psychosis develops.

Seizures can be amanaged with anitconvulsants but drug may ned to be discontinued.

Group V

Amoxicillin/Clav. acid

Clofazimine

Imipenem, Meropenem

Linezolid
Hypersensitivity, GI disturbances

Skin discolouration, GI disturbances

Hypersensitivity, neurotoxocity

Neuropathy, anaemia

For serious allergic reactions, stop all therapy pending, resolution of reaction.

Monitor blood count

Monitor blood count, avoid prolonged use. Stop if peripheral neuropathy or hematological problems occur.

$\underline{\text { Isoniazid (high dose) }}$ Peripheral neuropathy, hepatotoxicity Give with pyridoxine 
weighs the benefit expected from them. Each country should ensure an appropriate diagnostic capacity and good clinical and operational case management of all MDR-TB cases. To prevent misuse and avoid development of drug resistance, should be considered, limiting authorization for prescription of new drugs to registered physicians, well trained and experienced in the treatment of MDR-TB cases eligible for the new or repurposed drug, so as. Adequate laboratory facilities for second-line drug susceptibility testing should be in place.

\section{Healthcare system factors influenc- ing treatment results of patients} with multidrug-resistant tuberculosis

A recent European Centre for Disease Prevention and Control (ECDC) technical report, ${ }^{57}$ identified some healthcare system factors that are key to achieving good treatment results for patients with MDR-TB. The document suggests the following among actions to improve the success of treatment for MDR-TB patients in EU countries: timely diagnosis of resistant strains; development of European guidance on treatment regimen design; development of EU-wide criteria for hospitalization of MDR- and XDR-TB patients; implementation of a minimum package of cross-border MDR-TB control and care and creation of collaboration mechanisms to ensure a continuum of care between countries; reporting of treatment results; implementation of patient-centered approaches encompassing the social needs of MDR-TB patients; longterm sustainable financing strategies to ensure prevention, diagnosis, treatment and care for MDR-TB patients. Shared European criteria for hospitalization and discharge of MDR- and XDR-TB patients are needed so to rationalize the duration of hospitalization and a well-designed discharge plan, including social aspects of care, is a need to achieve a successful outcome.

\section{Rationale for regional multidrug- resistant tuberculosis referral centers in Italy and Hub \& Spoke model}

Italy is considered a low TB and MDR-TB incidence country; ECDC/WHO Regional Office for Europe reports 78 MDR-TB cases (3,3\% of cases with drug susceptibility test results) in 2013 , of which 7 were XDR cases. ${ }^{58}$ Note that in Italy Respiratory and Infectious Diseases Centers are more than twice as many MDR-TB cases. Thus in-patient MDR-TB treatment is possibly dispersed over many hospitals with clinicians hospitalizing and treating on average less than one case annually: this limited experience is likely to result in inappropriate clinical case management and emergence of resistance. As suggested by ECDC, concentration of in-patient MDR TB treatment could be considered in countries with low numbers of MDR TB patients. ${ }^{57}$ The identification of regional MDR-TB referral centers in our country would enable: i) concentration of in-patient MDR-TB treatment allows to provide more specialized care with appropriate clinical case management and to prevent emergence of drug resistance; ii) to facilitate monitoring and active pharmaco-vigilance, especially for new drugs, during ambulatory treatment after discharge; iii) to have a patient-centered support (social, economical, psychological), including treatment of co-morbidities, to ensure completion of treatment.

Referral regional areas of activity are based on the Hub \& Spoke model which will enhance and facilitate the coordination of care for those affected by MDR-TB, carers and their communities in managing the burden of disease. Aims of a TB Hub \& Spoke model are to enhance and improve care, reduce inequalities, improve education and achieve quality standards of care, and to set up regional and local meetings to review of complex cases /drug resistance and facilitate cohort review. Hub and spoke model is the preferred service model with all TB services able to provide standard case management and onward referral to the MDR-TB specialist hub of the network (multidisciplinary team with doctors, nurses, microbiologist, social worker, and voluntary sector involved) which can provide enhanced case management and ensure that all MDR-TB patients can access a level of care equal to their needs whilst ensuring that core delivery of care remains within their local services.

\section{References}

1. Diacon AH, Pym A, Grobusch M, et al. The diarylquinoline TMC207 for multidrugresistant tuberculosis. N Engl J Med 2009;360:2397-405.

2. Diacon AH, Donald PR, Pym A, et al. Randomized pilot trial of eight weeks of bedaquiline (TMC207) treatment for multidrug-resistant tuberculosis: long-term outcome, tolerability, and effect on emergence of drug resistance. Antimicrob Agents Chemother 2012;56:3271-6.

3. Diacon AH, Pym A, Grobusch MP, et al. Multidrug-resistant tuberculosis and culture conversion with bedaquiline. N Engl J Med 2014;371:723-32.

4. Ndjeka N, Conradie F, Schnippel K, et al. Treatment of drug-resistant tuberculosis with bedaquiline in a high HIV prevalence setting: an interim cohort analysis. Int $\mathrm{J}$ Tuberc Lung Dis 2015;19:979-85.

5. Guglielmetti L, Le Dû D, Jachym M, et al.
MDR-TB Management group of the French National Reference Center for Mycobacteria and the Physicians of the French MDR-TB Cohort. Compassionate use of bedaquiline for the treatment of multidrug-resistant and extensively drugresistant tuberculosis: interim analysis of a French cohort. Clin Infect Dis 2015;60:188-94.

6. Nunn AJ, Rusen I, Van Deun A, et al. Evaluation of a standardized treatment regimen of anti-tuberculosis drugs for patients with multi-drug-resistant tuberculosis (STREAM): study protocol for a randomized controlled trial. Trials 2014;15:353.

7. Gler MT, Skripconoka V, Sanchez-Garavito E, et al. Delamanid for multidrugresistant pulmonary tuberculosis. N Engl J Med 2012;366:2151-60.

8. Skripconoka V, Danilovits M, Pehme L, et al. Delamanid improves outcomes and reduces mortality for multidrug-resistant tuberculosis. Eur Respir J 2013;41:2393400.

9. Wells CD, Gupta R, Hittel N, Geiter LJ. Long-term mortality assessment of multidrug-resistant tuberculosis patients treated with delamanid. Eur Respir J 2015;45:1498-501.

10. World Health Organization. The use of bedaquiline in the treatment of multidrugresistant tuberculosis-Interim policy guidance. Geneva: WHO; 2013. WHO/HTM/ TB/2013.6.

11. World Health Organization. The use of delamanid in the treatment of multidrugresistant tuberculosis. Geneva: WHO; 2014. WHO/HTM/TB2014.23.

12. Matteelli A, D'Ambrosio L, Centis R, et al. Compassionate and optimum use of new tuberculosis drugs. Lancet Infect Dis 2015;15:1131-2.

13. Rustomjee R, Zumla A. Delamanid expanded access novel treatment of drug resistant tuberculosis. Infect Drug Resist 2015;8:359-66.

14. World Health Organization. Policy implementation package for new TB drug introduction. Geneva: WHO; 2014. WHO/HTM/ TB/2014.22

15. Tang S, Yao L, Hao X, et al. Clofazimine for the treatment of multidrug-resistant tuberculosis: prospective, multicenter, randomized controlled study in China. Clin Infect Dis 2015;60:1361-7.

16. Diacon AH, Dawson R, von GrooteBidlingmaier F, et al. Bactericidal activity of pyrazinamide and clofazimine alone and in combinations with pretomanid and bedaquiline. Am J Respir Crit Care Med 2015;191:943-53.

17. Sotgiu G, Centis R, D’Ambrosio L, et al. Efficacy, safety and tolerability of linezolid 
containing regimens in treating MDR-TB and XDR-TB: systematic review and metaanalysis. Eur Respir J 2012;40:1430-42.

18. Lee M, Lee J, Carrol MW, et al. Linezolid for treatment of chronic extensively drugresistant tuberculosis. $\mathrm{N}$ Engl $\mathrm{J}$ Med 2012;367:1508-18.

19. Tang S, Yao L, Hao X, et al. Efficacy, safety and tolerability of linezolid for the treatment of XDR-TB: a study in China. Eur Respir J 2015;45:161-70.

20. De Lorenzo S, Alffenaar JW, Sotgiu G, et al. Efficacy and safety of meropenem/clavulanate added to linezolid containing regimens in the treatment of M/XDR-TB. Eur Respir J 2013;41:1386-92.

21. Tiberi S, D'Ambrosio L, De Lorenzo S, et al. Ertapenem in the treatment of multidrugresistant tuberculosis: first clinical experience. Eur Respir J 2016;47:333-6.

22. Merle CS, Sismanidis C, Bah Sow 0, et al. A pivotal registrationphase III, multicenter, randomized tuberculosis controlled trial:design issues and lessons learnt from the Gatifloxacin for TB (OFLOTUB) project. Trials 2012;13:61.

23. Rustomjee R, Lienhardt C, Kanyok T, et al. A Phase II study of thesterilising activities of ofloxacin, gatifloxacin and moxifloxacin inpulmonary tuberculosis. Int $\mathrm{J}$ Tuberc Lung Dis 2008;12:128-38.

24. Jindani A, Harrison TS, Nunn AJ, et al. High-dose rifapentine with moxifloxacin for pulmonary tuberculosis. N Engl J Med 2014;371:1599-608.

25. Merle CS, Fielding K, Sow OB, et al. A fourmonth gatifloxacin-containingregimen for treating tuberculosis. N Engl J Med 2014;371:1588-98.

26. Gillespie SH, Crook AM, McHugh TD, et al. Four-month moxifloxacin-based regimens for drug-sensitive tuberculosis. N Engl J Med 2014; 371:1577-87.

27. Diacon AH, Dawson R, von GrooteBidlingmaier F, et al. 14-day bactericidal activity of PA-824, bedaquiline, pyrazinamide, and moxifloxacin combinations: a randomized trial. Lancet 2012;380:986-93.

28. Dawson R, Diacon AH, Everitt D, et al. Efficiency and safety of the combination of moxifloxacin, pretomanid (PA-824), and pyrazinamide during the first 8 weeks of antituberculosis treatment: a phase $2 \mathrm{~b}$,open-label, partly randomized trial in patients with drug-susceptible or drugresistant pulmonary tuberculosis. Lancet 2015;385:1738-47.

29. Daley CL, Caminero JA. Management of multidrug resistant tuberculosis. Semin Respir Crit Care Med 2013;34:44-59.

30. Johnston JC, Shahidi NC, Sadatsafavi M, Fitzgerald JM. Treatment outcomes of multidrug-resistant tuberculosis: a systematic review and meta-analysis. PLoS One 2009;4:e6914.

31. Orenstein EW, Basu S, Shah NS, et al. Treatment outcomes among patients with multidrug-resistant tuberculosis: systematic review and meta-analysis. Lancet 2009;9:153-61.

32. World Health Organization. WHO Companion handbook to the WHO guidelines for the programmatic management of drug-resistant tuberculosis. Geneva: WHO; 2014. WHO/HTM/TB/2014.11.

33. Lange C, Abubakar I, Alffenaar JW, et al. Management of patients with multidrugresistant/extensively drug-resistant tuberculosis in Europe: a TBNET consensus statement. Eur Respir J 2014;44:23-63.

34. Akçakır Y. Correlates of treatment outcomes of multidrug-resistant tuberculosis (MDR-TB): a systematic review and metaanalysis. Thesis Dissertation. Montreal, Canada, McGill University Department of Epidemiology, Statistics and Occupational Health; 2010.

35. Bastos ML, Hussain H, Weyer $\mathrm{K}$, et al. Treatment outcomes of patients with multidrug-resistant and extensively drugresistant tuberculosis according to drug susceptibility testing to first- and secondline drugs: an individual patient data meta-analysis. Clin Infect Dis 2014;59: 1364-74.

36. Velásquez GE, Becerra MC, Gelmanova IY, et al. Improving outcomes for multidrugresistant tuberculosis: aggressive regimens prevent treatment failure and death. Clin Infect Dis 2014;59:9-15.

37. World Health Organization. Policy guidance on drug susceptibility testing (DST) of second-line anti-tuberculosis drugs. Geneva: WHO; 2008. WHO/HTM/TB/ 2008.392.

38. Dooley KE, Mitnick CD, Ann DeGroote M, et al. Old drugs, new purpose: retooling existing drugs for optimized treatment of resistant tuberculosis. Clin Infect Dis 2012;55:572-81

39. Katiyar SK, Bihari S, Prakash S, et al. A randomized controlled trial of high-dose isoniazid adjuvant therapy for multidrugresistant tuberculosis. Int J Tuberc Lung Dis 2008;12:129-45.

40. Alangaden GJ, Kreiswirth BN, Aouad A, et al. Mechanism of resistance to amikacin and kanamycin in Mycobacterium tuberculosis. Antimicrob Agents Chemother 1998;42:1295-97.

41. Krüüner A, Jureen $P$, Levina $K$, Ghebremichael S, Hoffner S. Discordant resistance to kanamycin and amikacin in drug-resistant Mycobacteriumtuberculosis. Antimicrob Agents Chemother 2003;47:2971-3.

42. Migliori GB, Lange C, Girardi E, et al; SMIRA/TBNET Study Group.
Fluoroquinolones: are they essential to treat multidrug-resistant tuberculosis? Eur Respir J 2008;31:904-5.

43. Ziganshina LE, Squire SB. Fluoroquinolones for treating tuberculosis. Cochrane Database Syst Rev 2008;1:CD004795.

44. Stahlmann R, Lode H. Safety considerations of fluoroquinolones in the elderly. Drugs Aging 2010;27:193-209.

45. Caminero JA, Sotgiu G, Zumla A, Migliori GB. Best drug treatment for multidrugresistant and extensively drug-resistant tuberculosis. Lancet Infect Dis 2010;10:621-9.

46. Angel JH, Bhati AL, Devadatta S, et al. A controlled comparison of cycloserine plus ethionamide with cycloserine plus thiacetazone in patientswith active pulmonary tuberculosis despite prolonged previous chemotherapy. Tubercle 1963;44:215-24.

47. Mathys V, Wintjens R, Lefevre P, et al. Molecular genetics of paraaminosalicylic acid resistance in clinical isolates and spontaneous mutants of Mycobacteriumtuberculosis. Antimicrob Agents Chemother 2009;53:2100-9.

48. Zumla A, Chakaya J, Centis R, et al. Tuberculosis treatment and managementan update on treatment regimens, trials, new drugs, and adjunct therapies. Lancet Respir Med 2015;3:220-34.

49. Chang KC, Yew WW, Tam CM, Leung CC. WHO group 5 drugs and difficult multidrug-resistant tuberculosis: a systematic review with cohort analysis and metaanalysis. Antimicrob Agents Chemother 2013;57:4097-104.

50. World Health Organization. Management of MDR-TB: a field guide. Geneva: WHO; 2008. WHO/HTM/TB/2008.402a.

51. Van Deun A, Maug AKJ, Hamid Salim MA, et al. Short, highly effective and inexpensive standardized treatment of multi-drugresistant tuberculosis. Am J Respir Crit Care Med 2010;182:684-92.

52. Aung KJM, Van Deun A, Declercq E, et al. Successful 9-month Bangladesh regimen for multidrug-resistant tuberculosisamong over 500 consecutive patients. Int J Tuberc Lung Dis 2014;18;1180-7.

53. Piubello A, Harouna SH, Souleymane MB, et al. High cure rate for standardised short-course multidrug-resistant tuberculosis treatment in Niger: no relapses. Int $\mathrm{J}$ Tuberc Lung Dis 2014;18:1188-94.

54. Bassili, Fitzpatrick C, Qadeer E, et al. Review article: a systematic review of the effectiveness of hospital and ambulatorybased management of multidrug-resistant tuberculosis. Am J Trop Med Hyg 2013;89 :271-80.

55. Fitzpatrick C, Floyd K. A systematic review of the costant cost effectiveness of treat- 
ment for multidrug-resistant-tuberculosis. Pharmacoeconomics 2012;30:63-80.

56. European Centre for Disease Prevention and Control. Expert opinion on the introduction of new drugs for tuberculosis control in the EU/EEA. Stockholm: Centre for Disease Prevention and Control; 2015.
57. European Centre for Disease Prevention and Control. Technical report. Rapid risk assessment: healthcare system factors influencing treatment results of MDR TB patients. Stockholm: Centre for Disease Prevention and Control; 2014.

58. European Centre for Disease Prevention and Control/WHO Regional Office for Europe. Tuberculosis surveillance and monitoring in Europe 2015. Stockholm: European Centre for Disease Prevention and Control, 2015. 